\title{
Promoción del Desarrollo Económico Local (DEL): experiencias desde el turismo rural comunitario
}

\author{
Promotion of Local Economic Development (LED): \\ experiences from rural community tourism
}

Juan Rafael Hernández Navarro'

Amílkar Mora Sánchez ${ }^{2}$

Fecha de recepción: 20 de abril del 2012

Fecha de aprobación: 19 de agosto del 2012

Hernández, J; Mora, A. Promoción del Desarrollo Económico Local (DEL): experiencias desde el turismo rural comunitario.

Tecnología en Marcha. Vol. 25, № 46

Pág |04-1 I|

I Licenciado en Ciencias políticas, de la Universidad de Costa Rica, con maestría en Administración educativa, de la UNED, Costa Rica. Profesor e investigador de la Escuela de Ciencias Sociales del Tecnológico de Costa Rica, donde también fungió como coordinador de cátedras y proyectos de investigación. Ha sido profesor de la Maestría en Planificación de la Universidad Nacional, Costa Rica. Teléfono: (506) 2550-2562. Correo electrónico: jhernandez@itcrac.cr

2 Licenciado en sociología por la Universidad de Costa Rica, con Maestría en Desarrollo Económico Local, FLACSO CR-TEC. Profesor de la Escuela de Ciencias Sociales del Tecnológico de Costa Rica, actual coordinador de la Cátedra de Seminario de Estudios Costarricenses. Además, imparte cursos para la carrera de Gestión del Turismo Sostenible, del TEC. Teléfono: (506) 2550-20I3. Correo electrónico: amora@itcr.ac.cr 


\section{Palabras clave}

Desarrollo económico local, turismo rural comunitario, modelo de acompañamiento y desarrollo rural alternativo.

\section{Resumen}

Este artículo es una guía para la promoción del desarrollo económico local por medio del turismo rural comunitario. Se toma en cuenta una perspectiva teórico-metodológica para el acompañamiento a comunidades organizadas interesadas en la gestación de proyectos de turismo rural comunitario. La pretensión es compartir una metodología aplicada en comunidades rurales de Costa Rica que podría ser útil en situaciones similares.

\section{Key words}

Local economic development, community-based rural tourism, accompaniment and alternative rural development model.

\begin{abstract}
This article is a guide for the promotion of local economic development through community-based rural tourism. A methodological perspective for the accompaniment of organized communities interested in the development of community rural tourism projects will be taken into consideration. The claim is to share a methodology applied in rural communities of Costa Rica and that it might be useful in similar situations.
\end{abstract}

\section{Introducción}

Las tendencias globales de la economía han erosionado las capacidades endógenas de las comunidades en cuanto a planificar su desarrollo junto con los gobiernos locales y nacionales, cámaras empresariales, sindicatos, cooperativas, asociaciones de desarrollo y demás actores. En síntesis, la lógica de la globalización ha creado una dinámica económica de diseño exógeno y desde arriba; en un contexto de complicidad o desinterés de los gobiernos nacionales. En épocas anteriores -al menos en el caso de Costa Rica- dichos gobiernos procuraban conceder un gran protagonismo a la asociatividad local: los partidos políticos tenían una estrecha relación con el movimiento cooperativo, las asociaciones comunales de desarrollo, sindicatos, comités y una banca pública al servicio de esos proyectos'.

La misma dinámica económica se presenta en otros países,: se privilegian los centros urbanos por ser

I Hay que tener en cuenta que hasta 1995, merced a una nueva Ley Orgánica del Banco Central, el sistema bancario costarricense no admitía que bancos y sociedades financieras privadas pudiesen captar depósitos del público. Además, se les dio acceso al encaje bancario por la misma ley (Hidalgo, 2003, p.233). más desarrollados en infraestructura, se cuenta con más servicios y la población está más calificada, sin dejar de lado los recursos financieros a los cuales tiene acceso. De esta forma, las poblaciones urbanas marginales así como las rurales son los grandes excluidos: territorios usualmente dinámicos socioeconómicamente experimentan un proceso de rezago.

De esta forma se da un fraccionamiento de la economía: un segmento se articula exitosamente en las dinámicas globales, en tanto que el sector tradicional se estanca. Sin embargo, para grupo vinculado a las dinámicas globales lo hace dependiente del gran capital transnacional. Esta característica del sistema socioeconómico costarricense ha sido observada por Hidalgo (2003). Este autor hace notar que entre 1983 y el 2000 -que cubren el periodo de reformas hacia las condiciones actuales- el coeficiente de apertura $^{2}$ (dependencia) ha pasado de 47.7 a 103.0 (Hidalgo, 2003: 258s). En el cuadro I se muestra el significado actual de este dato.

2 "El cociente de la suma de las exportaciones y las importaciones de bienes y servicios entre el PIB" (Hidalgo, 2003, p.258). 


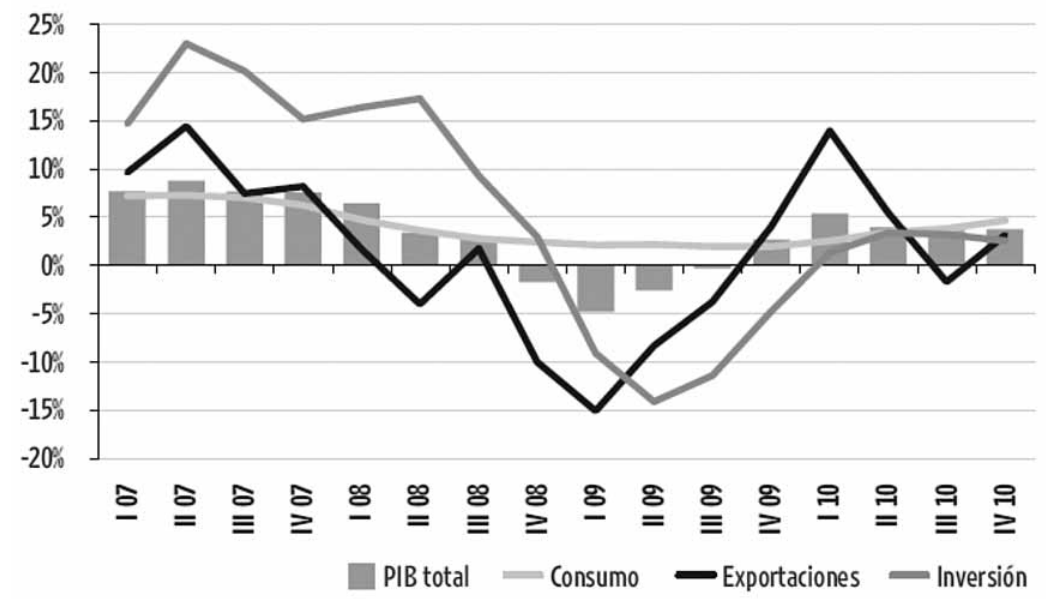

Cuadro I. Crecimiento real del PIB, el consumo, las exportaciones y la inversión (tasa de variación interanual por trimestres)

Fuente: Informe del Estado de la Nación (201 I), p..49.

El potenciamiento de lo local, como estrategia político- económica alternativa, (Martin \& Schumann, 1998) es viable en el contexto de la globalización, pues posibilita una articulación efectiva de las iniciativas locales, con las dinámicas globales.

Así, se parte del hecho que comunidades venidas a menos en esta coyuntura, con actividades económicas tradicionales, pueden fomentar emprendimientos exitosos, basados en sus prácticas ancestrales combinadas con nuevas actividades, de manera que cobren su valor diferenciado en el ámbito global. Hay casos exitosos de esta estrategia, en comunidades que han apostado al turismo rural comunitario, pues sus principios, lejos de lesionar las prácticas tradicionales, propician su perpetuación y las ponen en valor. A este tipo de planteamiento apunta el modelo expuesto más adelante.

\section{Justificación}

La historia político-económica de nuestras sociedades es de un centralismo abrumador. En Costa Rica, hablar de oportunidades, es hablar de la "Meseta Central", de la Gran Área Metropolitana3. Los secto-

3 La Gran Área Metropolitana (GAM) fue creada por el Instituto Nacional de Vivienda y Urbanismo (INVU) en 1982. Según Pujol (2003), fue creada como región, "pero no se creó una institucionalidad que la volviera una realidad política, aunque sí se creó un anillo de contención más allá del cual no se podía urbanizar". Se trata de una región metropolitana, en ella se res secundario y terciario, el destino de la inversión extranjera directa y de los planes de crecimiento y diversificación de los organismos públicos se han circunscrito, con contadas excepciones, a esta área geográfica. Lo anterior se acentúa con el ya citado problema de la globalización, que transfiere a la categoría de excluidos a muchos sectores ajenos a estos espacios.

Es en este contexto que se torna imperativa la valoración e implementación de estrategias de desarrollo económico local (DEL) que, con base en los recursos y capacidad endógena, permitan promover el "dinamismo económico y la mejoría en la calidad de vida de la población..." (Boisier, 2005, p. 52). En este sentido, se comparte en esta iniciativa la idea de S. Boisier, según la cual...

“...El desarrollo local dentro de la globalización es una resultante directa de la capacidad de los actores y de la sociedad local para estructurarse y movilizarse con base en sus potencialidades y en su matriz cultural, para definir, explorar sus prioridades y especificidades en la búsqueda de competitividad en un contexto de rápidas y profundas transformaciones" (Boisier, 2005, p. 52).

Como base de este planteamiento, se considera que la gestión del turismo rural comunitario puede

concentran los servicios públicos e institucionales, el gobierno y la mitad de la población del país (según el censo, 53\% en el 200l). 
convertirse en una herramienta eficaz de DEL, pues a esta estrategia de gestión del turismo, en el ámbito nacional, se le ha atribuido formalmente el objetivo de facilitar "que las personas habitantes de las comunidades rurales procuren la gestión de su propio desarrollo, incluido el manejo de destinos turísticos locales; además, que participen en la planificación y el aprovechamiento de los recursos naturales de su entorno de manera sostenible, a fin de que les permita una mejor condición de vida" (Ley de la República n 8724, "Fomento del Turismo Rural Comunitario", Art. I). En la práctica, se trata de iniciativas que exigen acompañamiento de organizaciones y expertos, además de simplificación de trámites. A esto se refiere Kyra Cruz (entrevistada por E. Cañada) cuando afirma que...

"La mayor parte de las iniciativas de turismo rural comunitario se encuentran en poblados de difícil acceso, donde existe únicamente una escuela. El promedio del nivel escolar de los empresarios de turismo rural comunitario es de sexto grado de primaria. Adicionalmente, muy pocos de ellos han podido ser turistas, por lo que se hace más difícil su interpretación de las necesidades de un viajero. Sin embargo, los empresarios de TRC generalmente son personas líderes en su comunidad, que pese a las limitaciones que enfrentan en cuanto a formación técnica, toman el riesgo y desarrollan iniciativas en este nuevo tipo de actividad para mejorar sus condiciones de vida y generar oportunidades de trabajo en sus comunidades" (Cañada, 2009, p.6).

La experiencia de los autores de este artículo en comunidades coincide en gran medida con lo anteriormente citado. El proceso estudiado se describe seguidamente:

a) Detección del interés en actividades de turismo y condiciones para el DEL

Es imperativo reconocer la ocurrencia de emprendimientos (exitosos o fallidos), así como la identidad socioeconómica de la comunidad y la idea de que es posible acceder a condiciones de desarrollo por esa vía (actividades agropecuarias, turísticas, agroindustriales, incluidas combinaciones de las mismas). Es decir, quienes procuran promover iniciativas de desarrollo local, no pueden pretender imponer su propia visión (externa) en determinada estrategia; es preciso partir del interés propio de los actores locales con sus propias percepciones e iniciativas.

b) Estrategia para modelar el proyecto comunitario de desarrollo

Se parte del análisis de las condiciones que favorecieron el éxito relativo de comunidades "exitosas" en actividades económicas afines (turismo, en el caso que nos ocupa). " para extrapolar estrategias y acciones exitosas en comunidades con condiciones análogas, pero ayunas de crecimiento en ese ámbito.

Se inicia con un análisis comparado de condiciones locales potencialmente favorables, con comunidades relativamente exitosas en actividades afines. En este caso, se inicia el establecimiento del paralelismo entre dos comunidades "exitosas"

c) Implementación de la estrategia

- Instrumentos: aplicación de técnicas de diagnóstico comunitario para valorar las características sociales, ambientales, de recursos (patrimonio social, natural y cultural)

- Dinámicas grupales ${ }^{4}$ para concienciación de la identidad comunal y definición del proyecto.

d) Aterrizar el resultado de la dinámica en una propuesta viable

a) La explicitación de una visión comunitaria de desarrollo, indispensable para la formulación de la propuesta.

b) Una declaración de valores, expresada en la carta del turismo ${ }^{5}$ (caso que nos ocupa) en que se configura el estilo de la participación comunitaria en turismo.

c) Acompañamiento a la asociación comunitaria de turismo, en el proceso de planificación estratégica.

d) Formalización: sensibilizar a la comunidad sobre la importancia de la institucionalización (cumplimiento de normas jurídicas y regla-

4 Dinámicas utilizadas: "La Telaraña" (Sales, 2000, p. 2),Ios "Mapas Parlantes" (PRODAF-GTZ, 1994, p.103) y el "Tiro al blanco" (Romero, s.f.: |4)

5 Véase: Carta del Turismo Sostenible. Conferencia mundial de turismo sostenible (Realizada en Lanzarote, Islas Canarias, España, abril 1995) 
mentos -salud, tributos, permisos- y dejar encaminado este proceso.

e) Cualificación en áreas afines (reservaciones, guianza, atención al cliente, alimentos y bebidas) lo anterior incluye mercadeo y accesibilidad con los clientes potenciales, v.gr. acompañamiento en el diseño de páginas web, para mostrarse en internet.

- Los productos tangibles que derivan de las acciones anteriores incluyen los siguientes:

Historias de las comunidades.

- Cartas del turismo de cada comunidad: producción de tres documentos editados y entregados formalmente en Providencia, San Jerónimo, Suretka (comunidades de los distritos de Copey de Dota, San Pedro de Pérez Zeledón, Bratsi y Amubri de Talamanca, respectivamente, los dos primeros de la provincia de San José y el último de la provincia de Limón).

- Inventarios de recursos turísticos: producción de tres documentos editados y entregados formalmente a las respectivas asociaciones de Providencia, San Jerónimo y a la de Talamanca.

- Guía de formalización: dos ediciones de un documento producido y entregado formalmente luego del taller respectivo, en Providencia, San Jerónimo y Talamanca.

- Realización de visitas recíprocas de los miembros de las asociaciones comunitarias de turismo a las otras comunidades: los pobladores de Providencia visitaron Talamanca en dos oportunidades y otras tantas, San Jerónimo. Los de Talamanca visitaron Providencia. Algunas personas de San Jerónimo lograron ir a Talamanca. El propósito: obtener conocimiento, intercambiar experiencias y promocionar las redes.

- Investigación y acompañamiento en diseño constructivo y de sitio, de albergues, con respeto por estilos y materiales constructivos, tradicionales de la comunidad indígena de Talamanca. Producto: Informe final de Práctica de Especialidad para optar por el grado académico de bachiller en Arquitectura y Urbanismo "Propuesta de diseño: Turismo
Rural Comunitario"; aprobado por la Escuela de Arquitectura y Urbanismo del TEC y devuelto a los interesados; a saber:

- Diecisiete láminas con diseños arquitectónicos correspondientes a cuatro emprendimientos en Talamanca y diseños genéricos para nuevos emprendimientos con estilos constructivos autóctonos de las comunidades indígenas.

- Construcción de cabañas en Providencia y en San Jerónimo, con el acompañamiento de los promotores universitarios.

\section{Método de trabajo}

El sustento metodológico de este planteamiento investigativo interdisciplinario se obtiene de la conjunción entre la tradición asociada a la investigación cualitativa con técnicas participativas (desarrolladas principalmente para trabajo en comunidades) con técnicas analíticas propias de los recientes estudios de planificación del turismo aplicados conforme el planteamiento conceptual del turismo alternativo. No se limitan las posibilidades locales de establecer procesos exitosos (lo que sí ocurre con los modelos tradicionales de turismo).

Por otra parte, para reseñar la fundamentación teórica, se indica en los documentos del proyecto que...

"Con respecto al papel del turismo en el desarrollo económico local, algunos autores apuntan a que el turismo es una de las tres actividades económicas que generan alguna articulación de las economías centroamericanas (y de algunas partes de México) con la economía global. Las otras son las emigraciones y la maquila. Sin embargo, sobre el tipo de integración que el turismo permite, algunos autores tienen una visión fundamentalmente optimista del turismo en relación con el tema del desarrollo, mientras que otros tienen una visión fundamentalmente pesimista. En este sentido, algunos estudios cuantitativos han mostrado que en aquellos países y regiones donde se ha dado una especialización en turismo, los indicadores económicos tienden a ser significativamente mejores que en aquellos en que no hay desarrollo del turismo. Estos estudios que tratan sobre las bondades y perjuicios del turismo, han olvidado explicar 
por qué razones las comunidades puede ser objeto de desarrollo turístico, dándolo por sentado. Las mayorías de las veces se intuye que hay razones aceptadas y que asocian este hecho a la expansión de la demanda turística, y conciben con ello que las comunidades tienen un papel poco proactivo. Menos aún existe preocupación en explicar por qué comunidades con sorprendentes similitudes socio-productivas tienen diferentes resultados en constituirse en destinos turísticos alternativos exitosos". (Hernández y Mora, 2009, p. 35).

El proceso, como se indicó, se desarrolla a partir de una metodología participativa en cada comunidad, para entender las razones que las llevaron a desarrollar turismo con éxito al igual que entender las razones de por qué otras comunidades no lo han logrado, a pesar de condiciones aparentemente similares.

Se pretende obtener una serie de insumos metodológicos prácticos, los cuales podrían ayudar en la construcción de una estrategia de apoyo a otras comunidades que potencialmente puedan ser hospederas de una estrategia de desarrollo sustentado en el turismo.

La metodología empleada en el proyecto privilegia la investigación cualitativa. De esta manera, técnicas como las de observación, la entrevista semiestructurada y las técnicas participativas grupales, resultaron centrales en las indagatorias de campo.

Para la recolección de información en la primera etapa, se utilizaron las técnicas de la entrevista y la observación. La primera se aplicó a un conjunto de informantes clave en cada comunidad; personas emprendedoras o pioneros, articuladores de la organización comunal con el sistema turístico internacional ${ }^{6}$.

Los trabajos correspondientes a la planificación del turismo comunitario (segunda etapa del proyecto FODELOTUR) en Providencia de Dota y San Jerónimo de Pérez Zeledón (ambas poblaciones rurales pertenecientes a la provincia de San José) se inician con un acercamiento basado en los pri-

6 El análisis de estas entrevistas se basó en el criterio de saturación, consistente en determinar los datos de interés según se van esclareciendo, al aparecer en ocasiones repetidas en cada entrevista. meros hallazgos de la aplicación de las técnicas de la primera etapa.

La primera etapa consistió en la identificación de los actores locales más activos, para posteriormente plantear una sesión abierta y convocar a todos los habitantes de cada comunidad para participar del Taller para la elaboración colectiva de la "Carta del Turismo". Esta carta -en la propuesta de planificación del turismo planteada en esta metodología- es la base axiológica de todas las acciones que la organización comunitaria para el turismo ha de emprender a mediano y largo plazo, en función de su desarrollo. Esta carta es una declaración de principios basada en la Carta de Lanzarote (1995) para un turismo sostenible (referencia digital en la bibliografía).

Se realizaron talleres comunitarios, para la confección de la Carta del Turismo de cada comunidad; en estos, se aplican técnicas participativas como la "dinámica de la Telaraña" (Sales, 2000:2), los “Mapas Parlantes" (PRODAF-GTZ, 1994: 103) y el "Tiro al blanco" (Romero, s.f.: I4) con las que se logra establecer y jerarquizar los principios identificados por los participantes como fundamentales para el desarrollo de su comunidad.

Posteriormente, se realiza el levantamiento del "Inventario de Recursos Turísticos" de cada comunidad. Su fundamento metodológico y ejecución en el campo se resumen a continuación:

“La razón de ser de un inventario del turismo es la de evaluar los recursos turísticos y de otros factores influyentes en el turismo en una zona determinada. Constituyen la base para decidir si una zona tiene potencial de desarrollo turístico o no. También indican el tipo y alcance de ese desarrollo. Si esta evaluación de "pre-viabilidad" es en general positiva e indica que el turismo puede desarrollarse de forma deseable, entonces la zona puede proceder a una planificación detallada" (Cfr. OMT, 1999).

"Se ha tratado de inventariar y categorizar los principales recursos turísticos con que cuenta la comunidad, tanto los recursos naturales, las inversiones en planta aprovechable para el turismo, así como las actividades económicas, servicios sociales, infraestructura y de organización de la comunidad. Este estudio, si la comunidad considera que debe seguirse adelante en la pla- 
nificación el turismo, llevará a la realización de un diagnóstico del turismo local, y que a su vez permita la planificación estratégica del turismo" (FODELOTUR, 2008).

Los respectivos inventarios se levantan por medio de fichas con un formato avalado por el Instituto Costarricense de Turismo (ICT). En ese proceso trabajaron conjuntamente los habitantes de cada comunidad y los estudiantes del programa de Bachillerato en Gestión del Turismo Sostenible adscrito a la Escuela de Ciencias Sociales.

El compromiso comunitario con los principios consignados en sus respectivas "Cartas del Turismo" y la síntesis de resultados de cada Inventario de Recursos Turísticos permiten el inicio de las gestiones de fortalecimiento de la densidad y calidad institucional expresada en el acompañamiento para la creación y posterior formalización de las asociaciones comunitarias para la gestión del turismo alternativo. Además, se estima necesario el acompañamiento en la gestión de financiamiento para infraestructura de hospedaje, materiales y equipamiento.

La siguiente etapa del proceso consistió en la impartición del Taller de Formalización. Para esto se elaboró una guía didáctica en forma de folleto ilustrado (tercer producto) que se distribuyó entre los asistentes a dicho taller. Fruto de lo anterior, se inicia el proceso de formalización. Se da el acompañamiento para la formalización de las asociaciones comunitarias de turismo (Aturena en San Jerónimo y ASOCATUR en Providencia y Dikivi'-Cámara de Comercio Turismo e Industria de Talamanca-) y de los diversos emprendimientos que los actores socioeconómicos (familias) locales han decidido realizar en función de su incursión en el turismo.

Además, en el segundo año del proyecto se manifestó gran interés por la construcción de cabañas para hospedaje. Asimismo, los investigadores brindan un acompañamiento a los actores de las comunidades que ya han asumido su proyecto. A estas alturas del proceso ya se cuenta con información obtenida de las indagatorias a profundidad realizadas en las dos comunidades "exitosas" en turismo rural comunitario (San Gerardo de Rivas, Pérez Zeledón, y San Gerardo de Dota).

7 Designación en lengua Bribri (significa río grande).
Las últimas tareas del proyecto en las comunidades fueron de dos tipos, hacia adentro y hacia afuera del entorno comunal: capacitación e incidencia sociopolítica. En lo primero se contó nuevamente con la valiosa participación de estudiantes avanzadas de Gestión del Turismo Sostenible, esta vez como asistentes especiales, bajo la tutela de los investigadores responsables del proyecto. Se prepararon e impartieron talleres de capacitación en calidad para el servicio turístico. Asimismo, profesores de la Escuela de Ciencias Sociales impartieron talleres de ética del turismo.

En segundo lugar, se realizaron esfuerzos para apoyar las asociaciones de turismo así como hacer insidencia en instituciones del Estado, bancos y gobiernos locales. Como muestra de lo logrado, se instaló una antena satelital y consecuente se estableció la telefonía fija e internet; servicios con que hasta entonces no contaban (caso de Providencia de Dota). También se trabajó en la cogestión comunitaria de los recursos turísticos de los Parques Nacionales (guianza y construcción de senderos y acceso a la infraestructura de dichos parques).

Por último, se impartió un taller de planificación estratégica a los dirigentes e integrantes de las asociaciones, con la intención de que contaran con suficientes herramientas para continuar avanzando en su proceso de desarrollo basado en el turismo comunitario lo cual proveyó de mayor autonomía a las comunidades una vez finalizado el proyecto.

\section{Conclusiones}

Sobre el modelo

El modelo descrito parte de la selección de comunidades con potencial para el TRC, pero que no han tenido éxito a pesar de disponer de recursos y atractivos iguales o superiores a sus homólogos exitosos. También se toma en cuenta el reconocer y valorar los factores determinantes así como tratar de extrapolarlos a las primeras regiones menos exitosas como son los casos de San Gerardo de Rivas de Pérez Zeledón y San Gerardo de Dota en comparación con sus contrapartes San Jerónimo de San Pedro de Pérez Zeledón y Providencia de Dota, las cuales sí han tenido éxito.

Para el caso de los distritos de Bratsi y Amubri, ubicados en el cantón de Talamanca en la provincia 
de Limón, hay comunidades indígenas con patrones culturales, climáticos, económicos (entre otros) muy diferentes a los anteriores; de manera que no se cuenta con una contraparte para la contrastación. Sin embargo, los factores valorados como determinantes en las primeras regiones exitosas en cuanto al proceso de promoción, actividad del turismo rural comunitario, gestación de institucionalidad a favor del TRC coadyuvaron en validar el modelo.

\section{Otros factores para complementar esta estrategia de desarrollo rural alternativa}

La asociatividad (capital social) es un valor esencial en proyectos como el propuesto en este artículo. Sin embargo, los emprendimientos individuales con capital financiero escaso para invertir en iniciativas no tradicionales sumado a la curva de aprendizaje de por medio hacen de estas empresas verdaderos actos de heroísmo, los cuales podrían ser más llevaderos con estrategias de economía social, tanto para el turismo, como para otras actividades complementarias.

El DEL mediante el turismo rural comunitario es novedoso y creativo. Siempre y cuando sea un conglomerado decididamente consolidado. Incluso así, no se debería abandonar las actividades tradicionales que hasta entonces habían constituido la base de su sustento socioeconómico. Es más, conviene poner en valor esas actividades a favor del turismo.

La universidad cumple un papel más allá de las actividades de investigación y promoción social: puede y debe brindar acompañamiento en la atención de necesidades detectadas como agroindustria. De esta forma se puede lograr un mayor valor agregado a productos propios de la zona (mora, café, industria láctea) y así no ser rehenes de los intermediarios con quienes negocian a precios ilógicos.

Por otro lado, el voluntariado de estudiantes y profesores es de un valor extraordinario. En las comunidades es valorado positivamente. Además, este tipo de voluntariado han abierto portillos para obtener apoyo en otras actividades complementarias.

Entes como los bancos, agencias de desarrollo y municipalidades constituyen factores muy sensibles. La denominada banca de desarrollo sigue estando en deuda con este tipo de emprendedores. Un patrón similar se detecta en las municipalidades en los casos analizados. En ocasiones se constata que los logros no se hacen gracias a la ayuda brindada por las municipalidades, sino que tiene éxito a pesar de no contar con ninguna ayuda. Justamente, la Ley de Fomento del Turismo Rural Comunitario pretende mejorar esta situación a favor de estas comunidades.

\section{Bibliografía}

Boissier, S. (2005). ¿Hay espacio para el desarrollo local en la globalización? En: Revista de la CEPAL, 86, agosto, p. 47-62.

Cañada, E. (2009). Legislar a favor del Turismo Rural Comunitario: el ejemplo de Costa Rica. Entrevista a Yorlenny Fontana, Kira Cruz y Olivier Pérez. Alba Sud. Opiniones en desarrollo, Programa Turismo Responsable. Disponible en www.albasud.org/publ/ docs/22.pdf

Hernández, J \& Mora, A (2008) Planteamiento proyecto FODELOTUR, Escuela de Ciencias Sociales, TEC.

Hernández, J \& Mora, A. (20|0). Informe Final. Proyecto FODELOTUR. CONARE. Instituto Tecnológico de Costa Rica.

Hidalgo, C \& Antonio, L. (2003). Costa Rica en evolución: política económica, desarrollo y cambio estructural del sistema socioeconómico costarricense (1980-2002). Editorial de la Universidad de Costa Rica - Publicaciones Universidad de Huelva. San José Costa Rica

Martin, H \& Schumann, H. (1998) La trampa de la globalización. Editorial Taurus, México.

PRODAF-GTZ (1994) Nuestro Congreso. Manual de diagnóstico rural participativo para la extensión campesina. Santiago de Puriscal. Costa Rica.

Programa Estado de la Nación. (201 I). Decimosétimo Informe Estado de la Nación en Desarrollo Humano Sostenible. CONARE - Defensoría de los Habitantes. Costa Rica.

Pujol, R. (2003). La estructura económica de San José y características del centro. En Pérez Mendoza, Salvador y Pujol Mesalles, Rosendo. Desafíos de los Centros de las Ciudades Mesoamericanas: los casos de tres metrópolis. Benemérita Universidad Autónoma de Puebla, FLACSO-Costa Rica, Grupo Interuniversitario de Montreal.

República de Costa Rica, Asamblea Legislativa. (2010). Ley de Fomento del Turismo Rural Comunitario.

Romero, P. (sf) Herramientas metodológicas participativas en proyectos de desarrollo.

Sales, M (2000) Dinámicas de grupo: técnicas de animación grupal. Universidad Champagnat. Buenos Aires, Argentina.

http://www.turismo-sostenible.org/docs/Carta-del-TurismoSostenible.pdf

http://www.emagister.com/curso-herramientas-metodologicasparticipativas-proyectos-desarrollo 